\title{
Hip orthopedic material presenting as an unusual rectal foreign body
}

\author{
Noelle Asmar*, Guillaume Perrod*, Gabriel Rahmi, Christophe Cellier
}

Université Paris Descartes Sorbonne Paris Cité, Assistance Publique-Hôpitaux de Paris

We report the case of an 80-year-old female patient referred to our hospital with suspicion of a rectal foreign body. Three days after undergoing right hip hemiarthroplasty, she started to experience lower crampy abdominal pain and aqueous non-bloody diarrhea. Her vital signs were normal. Physical examination revealed abdominal tenderness without guarding. Blood tests showed leukocytosis and raised inflammatory markers.

A pelvic X-ray showed a Steinmann pin that penetrated the pelvis. Computed tomography (CT) scan confirmed rectal perforation without signs of peritonitis (Fig. 1). A rectoscopy performed under $\mathrm{CO}_{2}$ insufflation disclosed a metal rod traversing the lower rectum and obstructing the lumen (Fig. 2). The mucosa was normal with no stigmata of hemorrhage.

Pin extraction was conducted through the same previous surgical approach. The patient was put on broad-spectrum antibiotics for one week despite negative cultures from the surgical site. A CT scan performed 3 days later showed fat stranding of the mesorectum but no abscess.

Usually, rectal foreign bodies are secondary to sexual practice, constipation or treatment of prolapsed hemorrhoids. However, post-surgical rectal foreign bodies have been described: an orthopedic Kirschner wire in a 74-year-old man one year after treatment for a left hip fracture, migration of a hemostatic Weck clip after laparoscopic prostatectomy, and several cases

Department of Gastroenterology and Digestive Endoscopy, Hôpital Européen Georges Pompidou, 20 rue Leblanc, 75015 Paris, France

Conflict of Interest:None

${ }^{\star}$ Co-first autorship

Correspondence to: Dr Guillaume Perrod, Department of Gastroenterology and Digestive Endoscopy, Hôpital Européen Georges Pompidou, 20 rue Leblanc, 75015 Paris, France, e-mail: guillaume.perrod@aphp.fr

Received 11 July 2018; accepted 26 July 2018;

published online 06 August 2018

DOI: https://doi.org/10.20524/aog.2018.0303

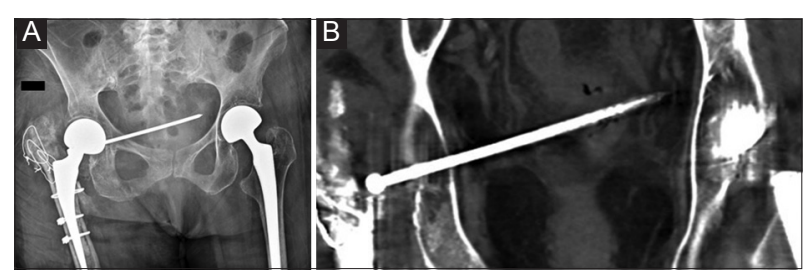

Figure 1 Radiograph of the pelvis (A) and computed tomography scan (B) with gap correction showing the Steinmann pin traversing he pelvis

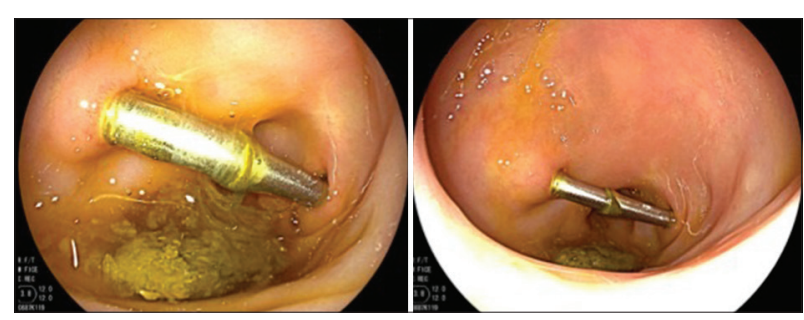

Figure 2 Rectal endoscopy showing a metal wire perforating the rectum side-to-side, and partially occluding the lumen

of perforated intrauterine contraceptive devices with rectal involvement [1-3]. In all cases, surgery combined with broadspectrum antibiotics was successful.

\section{References}

1. Yu FJ, Huang CJ, Hsieh JS. Unusual rectal bleeding caused by penetration of an intra-pelvic migrated guide pin. Dig Endosc 2013;25:469-470.

2. Patil A, Komanduri S. Weck clip migration into the rectum. Gastrointest Endosc 2012;75:426.

3. Eichengreen C, Landwehr H, Goldthwaite L, Tocce K. Rectal perforation with an intrauterine device: a case report. Contraception 2015;91:261-263. 